\title{
Fatigue crack growth in an aluminum alloy: Avalanches and coarse graining to growth laws
}

\author{
Ivan V. Lomakin $\odot,{ }^{1}$ Tero Mäkinen $\odot,{ }^{1,2, *}$ Kim Widell, ${ }^{3}$ Juha Savolainen $\odot,{ }^{1}$ \\ Sebastian Coffeng, ${ }^{1}$ Juha Koivisto ${ }^{\circ},{ }^{1}$ and Mikko J. Alava ${ }^{1,2}$ \\ ${ }^{1}$ Department of Applied Physics, Aalto University, P.O. Box 11000, 00076 Aalto, Espoo, Finland \\ ${ }^{2}$ NOMATEN Centre of Excellence, National Centre for Nuclear Research, A. Soltana 7, 05-400 Otwock-Świerk, Poland \\ ${ }^{3}$ Department of Mechanical Engineering, Aalto University, P.O. Box 11000, 00076 Aalto, Espoo, Finland
}

(Received 30 March 2021; revised 7 September 2021; accepted 21 October 2021; published 19 November 2021)

\begin{abstract}
In fatigue fracture the crack growth is slow and in many materials exhibits apparent self-similarity as expressed by the dependence of the growth velocity on a stress intensity factor that grows with the crack size. We study the intermittency of fatigue crack dynamics in aluminium alloys by optical tracking. A power-law distribution of crack tip jumps is found with an exponent close to two and a cutoff which increases with time or crack propagation. The cutoff is related to the crack velocity. We show how such a distribution evolves or coarse grains with the scale of observation or time window. The correlations of the crack propagation imply short-range memory effects in the underlying dynamics. Our results show universal features of fatigue cracks and how these lead to the crack growth and failure in material samples.
\end{abstract}

DOI: 10.1103/PhysRevResearch.3.L042029

\section{INTRODUCTION}

Fracture presents interesting fundamental science questions since the strength of materials as a concept has to deal with scales from the atomistic to the engineering of materials. One interesting everyday manifestation is fatigue failure, in which a load history is imposed on a sample (in the laboratory). The viewpoint from the materials science community is [1-4] that the main influence on the fatigue life and fatigue crack growth is the typical stress amplitude, for instance, during a loading cycle in a laboratory experiment. When the sample fails, the variability and the dependence on the details of the history are important and so is the impact of the material microstructure on these. Statistical physics has had an impact on fracture mechanics due to an understanding of the fluctuations in failure [5] such as in the depinning transition of cracks under applied loads [6-11] and creep dynamics as the thermally assisted motion of the crack tip [12-14]. Here, we take such a "crackling noise" [15] approach to fatigue crack growth (FCG). We show how the mesoscopic crack velocity arises from coarse graining the short-term and short-distance fluctuations in the dynamics. The crack tip jumps are widely distributed and the averaged growth law depends on the distribution and how it evolves with growth.

We next study the crack growth rate as described by crack growth laws such as the Paris-Erdogan one [16],

$$
\frac{d a}{d N}=C \Delta K^{m},
$$

\footnotetext{
*Corresponding author: tero.j.makinen@aalto.fi
}

Published by the American Physical Society under the terms of the Creative Commons Attribution 4.0 International license. Further distribution of this work must maintain attribution to the author(s) and the published article's title, journal citation, and DOI. where $a$ is the crack length, $N$ the number of cycles, $C$ a material- (and loading-) specific constant, $\Delta K$ the stress intensity factor (SIF) range, and $m$ the material- (and loading-) specific crack growth exponent. The SIF range can be written in terms of the peak SIF $\Delta K=(1-R) K_{\max }$ using the stress ratio $R=K_{\min } / K_{\max }$. Note that the peak stress plays only a minor part. For clarity the results presented here are expressed in terms of the peak SIF but this corresponds to only a horizontal shift in the Paris plots.

The apparent self-similarity [3,17] expressed by Eq. (1) may be misleading as a variety of more complex variants have been proposed, usually in terms of $\Delta K$ and $R$. The two main reasons for this complexity are the history or memory effects arising from crack closure [18] and the fact that the fracture process zone encodes the extent of plastic deformation due to the peak stresses during the fatigue process. Our work concentrates on the stochastic nature of FCG: We investigate the dynamics on timescales (or cycles) shorter than on which selfsimilar growth laws are formulated. This in itself presents a different look on fatigue fracture as a stochastic phenomenon with fluctuations [19].

Our approach is based on performing typical FCG experiments and following the development of the crack length optically (see Methods). This allows us to accomplish two goals. One is the determination of the "jumps" or steps that the crack length makes depending on the measurement time. Analogous data with a high resolution may be derived from acoustic emission (AE) studies [20-22], but the AE method is only an indirect measure whereas crack length measurements connect directly to the SIF.

The second step is the analysis of the scaling in the vein of Eq. (1) and its relation to the underlying dynamics. The main results that we obtain thus are threefold. First, a step size distribution is of power-law form with an exponent that differs from the usual values. Second, there is a slowly 
(a)

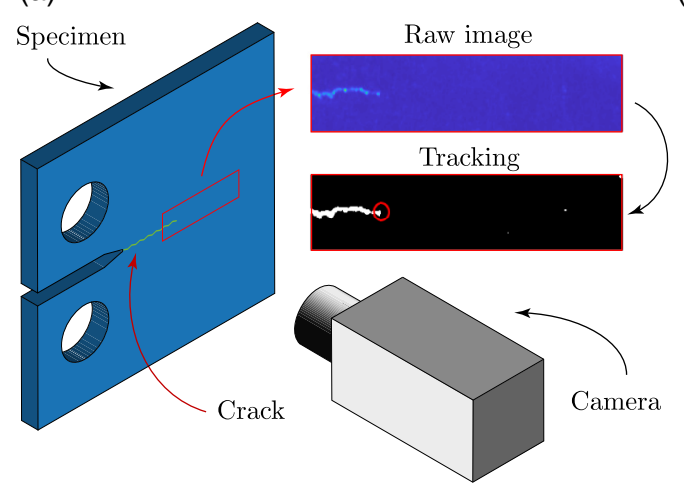

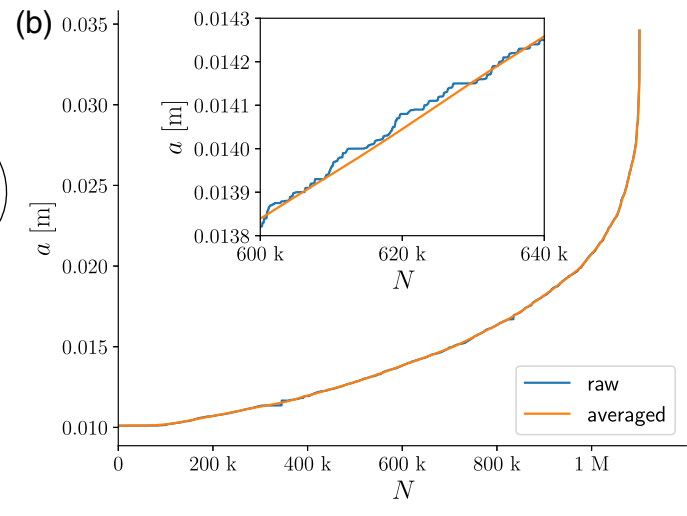

FIG. 1. (a) A schematic drawing of the experimental setup where the crack growth is monitored with a camera. The crack tip position is determined from the images by binarizing them and taking the edge pixel of the binarized area to correspond to the crack position. (b) The crack length $a$ resulting from the crack tip tracking as a function of cycle number $N$ (blue line). This jumpy raw signal is averaged to produce the smooth signal (orange line) used in the Paris plot. The inset shows a zoomed-in view of the two signals, highlighting the jumpy behavior.

increasing cutoff. Third, the dynamics exhibit short-range correlations.

\section{METHODS}

A commercially pure aluminum 1050 alloy was used in this study. Plates of thickness $5 \mathrm{~mm}$ were received from Alumeco Ltd. in strain-hardened and partially annealed condition corresponding to $\mathrm{H} 24$ temper. Specimens were polished using a final Struers DP suspension with 3- $\mu \mathrm{m}$ particles. For FCG tests standard compact tension specimens were produced using electrical discharge machining [Fig. 1(a)] so that the crack propagation occurs in the direction normal to the rolling direction. A MTS 858 hydraulic fatigue testing machine was used to load the samples using alternating force with a sinusoidal waveform and $10 \mathrm{~Hz}$ frequency. First, three types of tests were performed to test the effect of stress ratio $R$ (fixed $F_{\max }=1.5$ $\mathrm{kN}$ with $R$ values $0.1,0.3$ and 0.5 ). Additionally, tests with $F_{\max }=1.3 \mathrm{kN}$ and $R=0.1$ were performed to study the effect of the peak force with constant $R$. The tests were performed as described by the ASTM E647 standard [23] and the SIF determined accordingly.

To carry out crack propagation experiments the following procedure and data processing scheme was developed. The experimental setup consisted of a Canon EOS R digital camera imaging the sample every $4 \mathrm{~s}$ using a Canon MP-E 65-mm macrolens equipped with a ring light-emitting diode (LED) lamp. Uniform direct light created by the ring lamp gets deflected by the deformed surface in close proximity to the crack, illuminating it. We define the crack tip as the edge pixel of a contrast region on the binarized area in the vicinity of the visible crack tip using averaged local background intensity as the threshold value. To take the effect of specimen shift into account, the crack tip position was extracted relative to a stationary point on the specimen's surface. The accuracy of such an approach is of the order of one pixel and therefore comes down to image resolution $(6720 \times 4480$ pixels in the present work), and was equal to $4.5 \mu \mathrm{m}$ per pixel (or $112 \mathrm{~nm}$ per cycle, when the jumps are smeared over the whole imaging interval spanning 40 cycles). Such an approach is able to provide visual crack length tracking with an accuracy higher than the electric potential difference technique which is limited to $9 \mu \mathrm{m}$ for titanium (300 $\mu \mathrm{m}$ for aluminum) [23]. We apply this procedure sequentially to every image and additionally require the crack length to increase monotonously. This is done by constructing a monotonously increasing upper and lower envelope of the crack length signal and taking their average to correspond to the true crack length. This requirement can produce crack advancement jumps less than $100 \mathrm{~nm}$ per cycle but we have excluded such jumps from further analysis.

The estimation of the exponent $\gamma$ and the cutoff $\Delta a_{0}$ from the distributions was done by a maximum likelihood estimation [24]. First, the value of the exponent $\gamma$ was estimated by computing the maximum likelihood for a pure power law $p(\Delta a) \propto \Delta a^{-\gamma}$ by considering the $R=0.1$ and $R=0.3$ cases where the cutoff is high enough for the power-law region to be clearly visible (the estimation was done in a region excluding the cutoff). This yields an exponent $\gamma=2.0 \pm 0.3$. After that a maximum likelihood estimate was computed for the cutoff [for distribution of the form of Eq. (2)], using a fixed value $\gamma=2.0$ for the exponent. A different shape for the cutoff was considered by varying the exponent $\phi$ in the more general $p(\Delta a) \propto \Delta a^{-\gamma} \exp \left[-\left(\Delta a / \Delta a_{0}\right)^{\phi}\right]$ but when considering all the different cases, the best fit was found with $\phi$ close to unity.

\section{RESULTS}

By visually observing the crack advancement during an experiment [see Fig. 1(a) and Methods for details] we see that the crack advancement is not smooth but occurs in intermittent jumps [see Fig. 1(b)]. This jumpy crack position signal $a$ can be coarse grained by applying averaging schemes, which yield the smooth crack velocity $\frac{d a}{d N}$ (which is just the average crack advancement per cycle) used in Paris plots [see Fig. 2(a)].

Another direction would be to study these crack advancement jumps. Here, a jump $\Delta a$ is defined as the advancement of the crack between two images divided by the number of cycles between these images. This smearing of the observed jumps over the time interval between images serves just to compare the jump sizes with the coarse-grained crack velocity. We emphasize this difference between jumps in the crack position signal $\Delta a$ and the coarse-grained crack velocity $\frac{d a}{d N}$ by using 

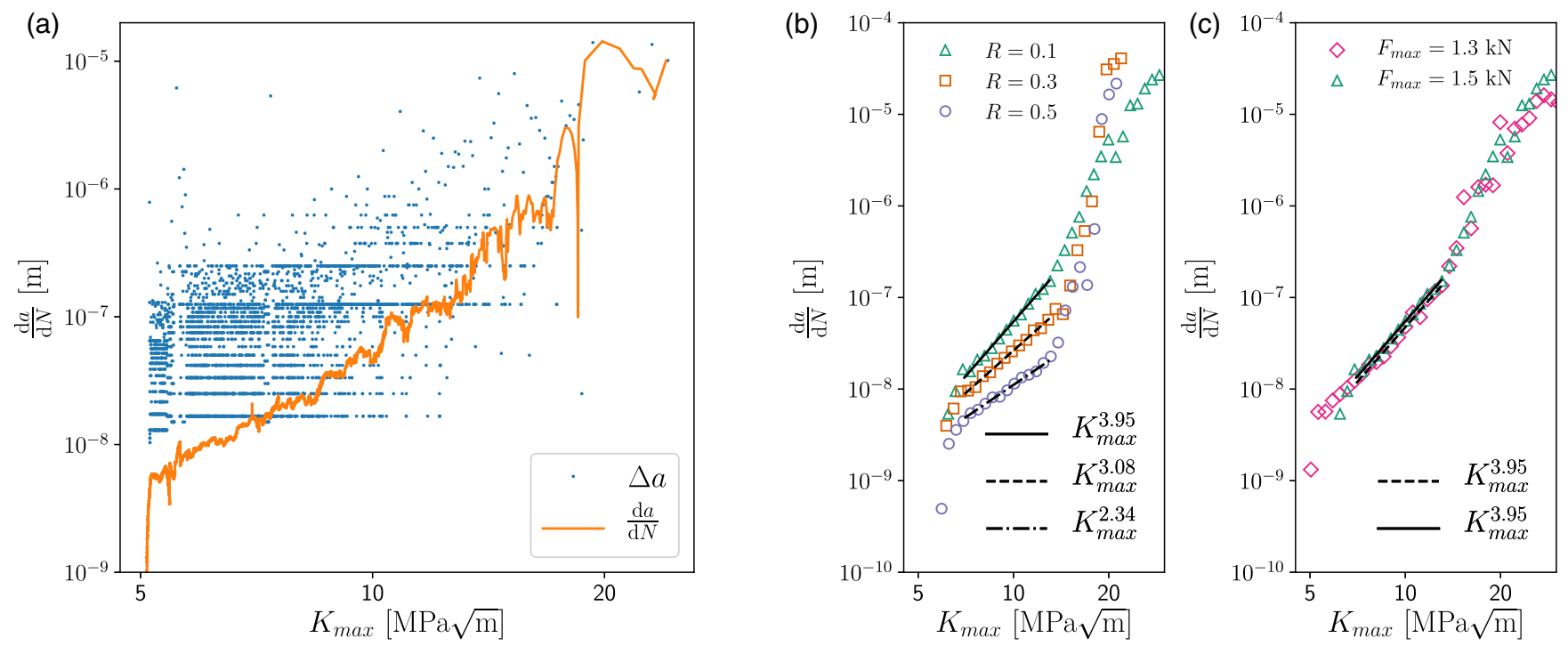

FIG. 2. (a) The crack advancement per cycle $\frac{d a}{d N}$ as a function of the peak SIF $K_{\max }$. The blue dots correspond to the jumps $\Delta a$ in the crack position signal and the orange line represents the coarse-grained crack velocity $\frac{d a}{d N}$. (b) The Paris curves (crack velocity $\frac{d a}{d N}$ vs the peak SIF $K_{\max }$ ) for three different values of stress ratio $R$. The power-law fits (black lines) around the region where the Paris-Erdogan law holds ( $K_{\max }$ from 7 to $13 \mathrm{MPa} \sqrt{\mathrm{m}}$ ) show that the exponent $m$ decreases with increasing $R$. (c) The Paris curves for two different values of $F_{\max }$. The power-law fits (black lines) around the same region as in (b) show that the exponent $m$ is constant and the prefactor $C$ increases very slightly with increasing $F_{\max }$.

different notations. Figure 2(a) shows this difference for a single experiment as a function of $K_{\max }$. The Paris curve $\frac{d a}{d N}$ (orange line) results from the crack advancement jumps $\Delta a$ (blue dots) via coarse graining. One should note that the lower values of $\frac{d a}{d N}$ compared to $\Delta a$ are due to the intermittency of the jumps (sometimes no crack advancement is detected).

The traditional analysis of the Paris curves shown in Figs. 2(b) and 2(c) reveals the effects of $R$ and $F_{\max }$ in this alloy. The region where the Paris-Erdogan law [Eq. (1)] holds corresponds to roughly $K_{\max }$ from 7 to $13 \mathrm{MPa} \sqrt{\mathrm{m}}$ and a power-law fit in this region reveals two things. First, the exponent $m$ decreases clearly (from 3.95 to 2.34) as $R$ increases from 0.1 to 0.5 . Second, as $F_{\max }$ increases from 1.3 to $1.5 \mathrm{kN}$ the exponent $m$ stays constant (fits give 3.95) but there is a tiny increase in the prefactor $C$. These values are typical for aluminum alloys, where $m$ is usually slightly over three [25-27] but sometimes smaller [28]. We also note that the usual models [29-31] of the effect of $R$ only apply a shift in the SIF term and do not change the slope in the Paris plots and therefore are not effective here.

Now we can take a crackling noise approach to the crack advancement jumps. By taking the aforementioned region where the Paris-Erdogan law holds and observing the probability density of $\Delta a$ in this region [Figs. 3(a) and 3(b)] we see that the distributions follow a power law $p(\Delta a) \propto \Delta a^{-\gamma}$ with an exponent $\gamma \approx 2$ for around two decades of jump sizes. This power-law distribution is also visible when one considers the region before this Paris-Erdogan regime. There is also a cutoff at high values of $\Delta a$ so the full probability density can be written as

$$
p(\Delta a) \propto \Delta a^{-\gamma} \exp \left(-\frac{\Delta a}{\Delta a_{0}}\right),
$$

where $\Delta a_{0}$ indicates the cutoff scale.
One can then fit this type of distribution to the data using Eq. (2) with $\gamma=2$ and $\Delta a_{0}$ as the free parameter (see Methods for details). For the case of different values of $R$ the probability density distributions with the fitted lines can be seen in Fig. 3(a). An increase in $R$ clearly corresponds to a
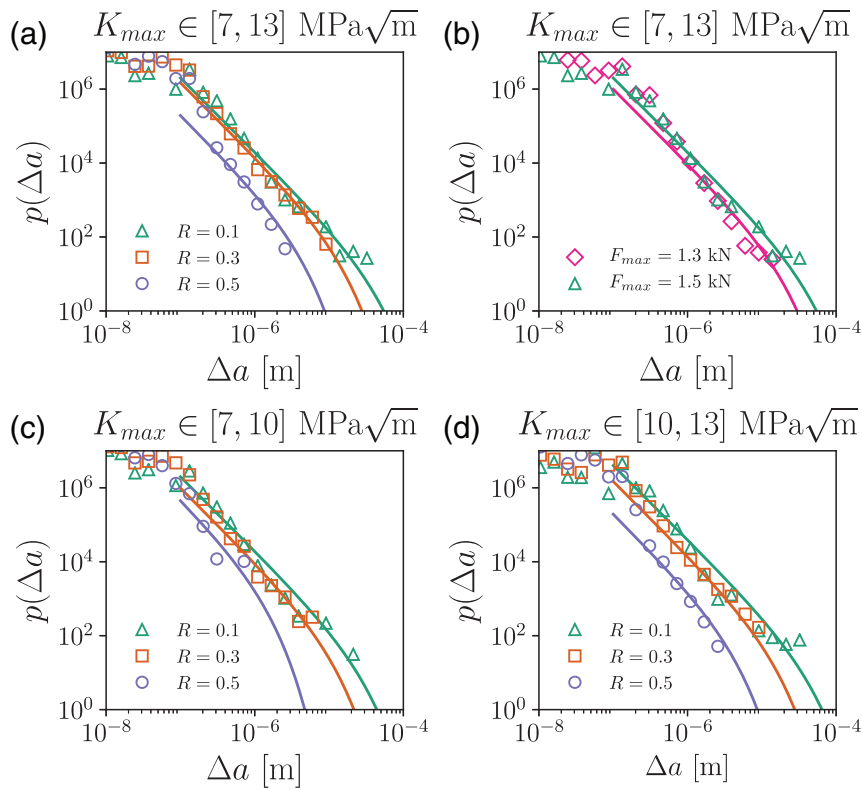

FIG. 3. (a) The probability density $p(\Delta a)$ of crack advancement jumps $\Delta a$, in the region where the Paris-Erdogan law [Eq. (1)] holds, for three different values of $R$. The lines correspond to fits of the cutoff size $\Delta a_{0}$ using Eq. (2) with $\gamma=2$. (b) Same as (a) but for two different values of $F_{\max }$ with $R=0.1$. (c) Same as (a) but only for the first half of the region $\left(K_{\max } \leqslant 10 \mathrm{MPa} \sqrt{\mathrm{m}}\right)$. (d) Same as (a) but only for the second half of the region $\left(K_{\max } \geqslant 10 \mathrm{MPa} \sqrt{\mathrm{m}}\right)$. 

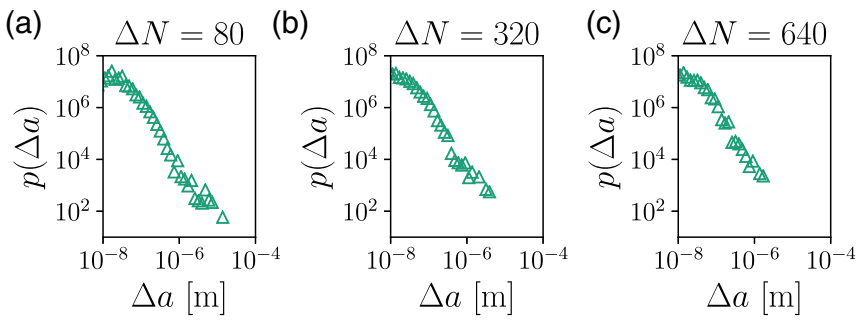

FIG. 4. The coarse-grained distributions of jump sizes (per cycle) after (a) one decimation, (b) three decimations, and (c) four decimations for the case $R=0.1$ and $F_{\max }=1.5 \mathrm{kN}$. Here, decimation refers to the rescaling of the time resolution (number of cycles between images $\Delta N$ ) by a factor of 2 .

decrease in the cutoff size. Similarly in Fig. 3(b) one can see this effect for the different values of $F_{\max }$. An increase in $F_{\max }$ corresponds to an increase in the cutoff size. Note that the number of events in this kind of statistics per experiment is constrained by the distance that the crack propagates in the power-law growth regime and the average step size-and for broad distributions the latter tends to be relatively large.

As the crack growth exponents $m$ differ between different values of $R$, the difference in the cutoff sizes should become more prominent as the SIF increases. We study this by splitting the Paris-Erdogan region in half (so $K_{\max }$ from 7 to $10 \mathrm{MPa} \sqrt{\mathrm{m}}$ and from 10 to $13 \mathrm{MPa} \sqrt{\mathrm{m}}$ ) and looking at them separately. As expected, the cutoff sizes in the first half [Fig. 3(c)] are smaller than in the second half [Fig. 3(d)] and the difference between the cutoffs for different $R$ increases in the second half.

The coarse graining that happens when one considers the crack advancement on larger and larger scales is demonstrated in Fig. 4 where the time resolution of the experiments is rescaled from the original number of cycles between images $\Delta N=40$. This decimation is done by interpolating the crack length data to a new grid of $N$ values with a different spacing (Fig. 4 shows this for $\Delta N=80, \Delta N=320$, and $\Delta N=640$, so after one, three, and four decimations-doublings of the cycles between images). The change of the distribution as $\Delta N$ increases shows that the self-averaging hides the larger crack tip jumps (when considering the jumps smeared over the whole imaging time window).

The behavior of $\Delta a_{0}$ we observe corresponds to the increase in the cutoff as the crack velocity increases, both in the case of differing $R$ and differing $F_{\max }$. This behavior can be quantified by taking the cutoff size determined for each of the distributions in Fig. 3 and plotting it against the average crack velocity $\left\langle\frac{d a}{d N}\right\rangle$ in the corresponding region of the Paris curve [Fig. 5(a)]. There is a clear correlation between these cutoffs determined from the distributions of the jump sizes and the averaged velocities determined from the coarse-grained signal. As a guide to the eye we plot a $\sqrt{\Delta a_{0}}$ dependence. Correlating the $\left\langle\frac{d a}{d N}\right\rangle$ against the average step size would give a too weak dependence. Thus we find in Fig. 5(b) that the cutoff has a direct correlation with the exponent $m$. The fatigue protocol$R$ value-relation to this is shown in Fig. 5(c), where we see a linear relationship, and thus of course the larger the $R$, the smaller is the $m$. Thus in the "creep limit" $R \rightarrow 1$ the crack velocity would tend to a constant.
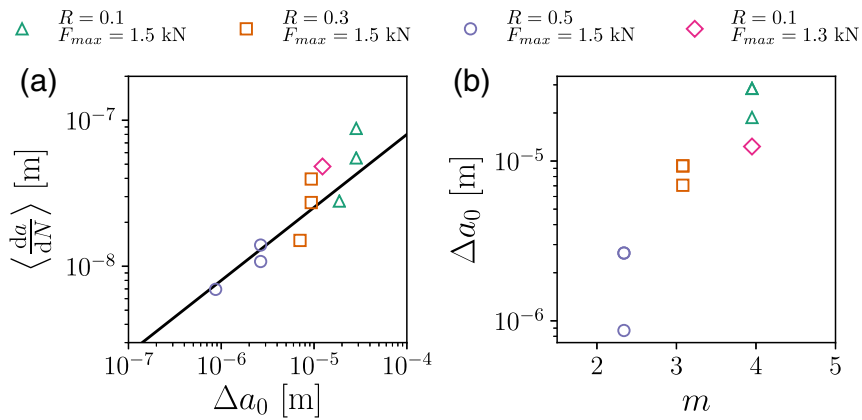

(c)
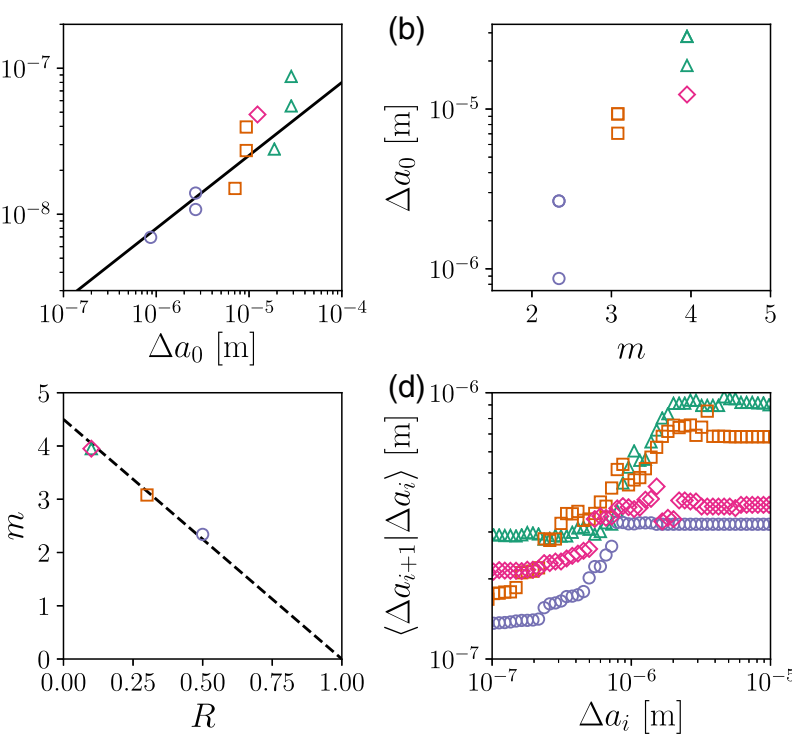

FIG. 5. (a) The cutoff size of the jump distributions $\Delta a_{0}$ plotted against the average crack velocity $\left\langle\frac{d a}{d N}\right\rangle$ in the corresponding regions of the Paris curves. The line is a square-root dependence. (b) The cutoff size of the jump distributions $\Delta a_{0}$ plotted against the ParisErdogan law exponent $m$. (c) The Paris-Erdogan law exponent $m$ as a function of $R$. (d) The conditional average of the subsequent jump size $\Delta a_{i+1}$ given the size of the previous jump $\Delta a_{i}$ as a function of the size of the previous jump. The averages are calculated in bins of at least 50 jumps.

Looking more closely at the distributions in Fig. 3 reveals that in addition to the cutoff behavior there is a slight excess of larger jumps. This can be seen as a bump visible in the distributions around $\Delta a=1 \mu \mathrm{m}$ which has previously been suspected [32] to arise from summing of several jumps into one. Here, we offer another explanation by looking at the correlations of subsequent jumps. One can compute the conditional average of a size of a jump $\Delta a_{i+1}$ given the size of the previous jump $\Delta a_{i}$, which is notated as $\left\langle\Delta a_{i+1} \mid \Delta a_{i}\right\rangle$ [see Fig. 5(d)]. Here, the averaging is done in (overlapping) bins centered at $\Delta a_{i}$ and the width of the bins is chosen so that each bin contains at least 50 jumps. All of the curves increase at a point corresponding to the location of the bump in the distributions (Fig. 3) and before and after this value the value is roughly constant. This would imply a presence of a memory effect: Small jumps tend to be followed right after by small jumps and large jumps $(\Delta a>1 \mu \mathrm{m})$ tend to be followed right after by larger jumps. These "large" jumps are however smaller compared to the previous, large one.

\section{CONCLUSIONS}

What we find when following in detail the fatigue crack growth is that the crack tip undergoes intermittent motion. These steps have a size distribution which is characterized by a particular power-law exponent, roughly two, with an increasing cutoff. The dynamics appears thus "critical" but the exponent value has no theoretical comparison in models of crack propagation in brittle materials $[5,11,33]$, where the 
theory of depinning transitions is applied to crack fronts. The typical avalanche size exponents are in fact limited above by a value of two in such theories even for elastic manifolds with long-range elasticity or interactions. Such coarse-grained theory includes mechanics (of elastic fields) and the material properties via a frozen, time-independent disorder field and is at best able to give predictions about crack fluctuations and creep rates in the limit of slow crack growth.

Here, we do not observe directly the avalanches along the fatigue crack in space and time, or the jumps in the average position of the crack with time. Instead, we follow the resulting integrated crack advancement over windows of time. The exponent value as such makes one expect that the mean step size has a weak dependence on the cutoff, contrary to such cases where the avalanche exponent is clearly smaller than two. The experimentally found cutoff increases with the crack advancement, and thus the step size and the typical crack growth rate averaged over longer times. This then relates the size distribution evolution to the "crack growth law," the Paris-Erdogan law [Figs. 5(a) and 5(b)]. This is often the most important part in FCG in that it terminates in rapid crack growth and thus is crucial for the sample lifetime.

On short scales the dynamics exhibit correlations such that they seem to imply pinninglike dynamics but with time and crack growth these get washed out, in analogy to the "average Paris law"-like dependence of the velocity on $\Delta K$ which in the spirit assumes Markovian, memoryless dynamics. The fluctuating character of the crack advancement implies, likewise, that the intrinsic noise or the fluctuations of the velocity depend on the timescale of observation.

The importance of our work is in that it shows that slow fatigue crack growth, typical of materials, follows from averaging over an intermittent dynamics. How quickly a crack grows in the self-similar regime of crack growth depends on how quickly the cutoff scale of the crack avalanches or jumps grows. This is then the direction for understanding how materials can made more resistant and how the loading history leads to faster growth [Figs. 5(b) and 5(c)]. The "material parameters" in the Paris-Erdogan self-similar growth law (or its relatives) such as the prefactor $C$ and the power-law exponent $m$ follow directly from the dependence of the crack statistics on the SIF. This dependence encodes the impact of the fatigue cycle details on how big jumps may happen and thus in particular on the exponent $m$.

Several questions and further directions are left. What is the origin or theoretical explanation of the statistical properties we find? Does the apparent intermittency follow universality, in particular in terms of the exponent of the distribution from one material to another? In summary, we expect that the fluctuations in fatigue failure in general and around the behaviors summed up by empirical growth laws are due to the small-scale crack avalanches. This presents fundamental questions about how to model fatigue failure, how predictable it is, and the role of such fluctuations in general.

\section{ACKNOWLEDGMENTS}

M.J.A. acknowledges support from the Academy of Finland (Center of Excellence program, 278367 and 317464) and the European Union Horizon 2020 research and innovation programme under Grant Agreement No. 857470 and from European Regional Development Fund via Foundation for Polish Science International Research Agenda PLUS programme Grant No. MAB PLUS/2018/8. J.K. acknowledges the funding from Academy of Finland (308235) and Business Finland (211715). The authors acknowledge the computational resources provided by the Aalto University School of Science "Science-IT" project.
[1] S. Suresh, Fatigue of Materials (Cambridge University Press, Cambridge, UK, 1998).

[2] W. Schütz, A history of fatigue, Eng. Fract. Mech. 54, 263 (1996).

[3] R. O. Ritchie, Incomplete self-similarity and fatigue-crack growth, Int. J. Fract. 132, 197 (2005).

[4] P. Chowdhury and H. Sehitoglu, Mechanisms of fatigue crack growth - a critical digest of theoretical developments, Fatigue Fract. Eng. Mater. Struct. 39, 652 (2016).

[5] M. J. Alava, P. K. Nukala, and S. Zapperi, Statistical models of fracture, Adv. Phys. 55, 349 (2006).

[6] J. Koivisto, J. Rosti, and M. J. Alava, Creep of a Fracture Line in Paper Peeling, Phys. Rev. Lett. 99, 145504 (2007).

[7] D. Bonamy, S. Santucci, and L. Ponson, Crackling Dynamics in Material Failure as the Signature of a Self-Organized Dynamic Phase Transition, Phys. Rev. Lett. 101, 045501 (2008).

[8] L. Ponson, Depinning Transition in the Failure of Inhomogeneous Brittle Materials, Phys. Rev. Lett. 103, 055501 (2009).

[9] L. Laurson, S. Santucci, and S. Zapperi, Avalanches and clusters in planar crack front propagation, Phys. Rev. E 81, 046116 (2010).
[10] J. Chopin, A. Bhaskar, A. Jog, and L. Ponson, Depinning Dynamics of Crack Fronts, Phys. Rev. Lett. 121, 235501 (2018).

[11] J. Bares, M. Barlet, C.-L. Rountree, L. Barbier, and D. Bonamy, Nominally brittle cracks in inhomogeneous solids: From microstructural disorder to continuum-level scale, Front. Phys. 2, 70 (2014)

[12] O. Lengliné, R. Toussaint, J. Schmittbuhl, J. E. Elkhoury, J. P. Ampuero, K. T. Tallakstad, S. Santucci, and K. J. Måløy, Average crack-front velocity during subcritical fracture propagation in a heterogeneous medium, Phys. Rev. E 84, 036104 (2011).

[13] K. T. Tallakstad, R. Toussaint, S. Santucci, J. Schmittbuhl, and K. J. Måløy, Local dynamics of a randomly pinned crack front during creep and forced propagation: An experimental study, Phys. Rev. E 83, 046108 (2011).

[14] T. Vincent-Dospital, A. Cochard, S. Santucci, K. J. Måløy, and R. Toussaint, Thermally activated intermittent dynamics of creeping crack fronts along disordered interfaces, Sci. Rep. 11, 1 (2021).

[15] J. P. Sethna, K. A. Dahmen, and C. R. Myers, Crackling noise, Nature (London) 410, 242 (2001).

[16] P. Paris and F. Erdogan, A critical analysis of crack propagation laws, J. Basic Eng. 85, 528 (1963). 
[17] A. P. Vieira, J. S. Andrade, Jr., and H. J. Herrmann, Subcritical Crack Growth: The Microscopic Origin of Paris Law, Phys. Rev. Lett. 100, 195503 (2008).

[18] W. Elber, Fatigue crack closure under cyclic tension, Eng. Fract. Mech. 2, 37 (1970).

[19] W. F. Wu and C. C. Ni, Statistical aspects of some fatigue crack growth data, Eng. Fract. Mech. 74, 2952 (2007).

[20] S. Deschanel, W. Ben Rhouma, and J. Weiss, Acoustic emission multiplets as early warnings of fatigue failure in metallic materials, Sci. Rep. 7, 13680 (2017).

[21] J. Weiss, W. Ben Rhouma, S. Deschanel, and L. Truskinovsky, Plastic intermittency during cyclic loading: From dislocation patterning to microcrack initiation, Phys. Rev. Materials 3, 023603 (2019).

[22] S. Shrestha, M. Kannan, G. N. Morscher, M. J. Presby, and S. M. Razavi, In-situ fatigue life analysis by modal acoustic emission, direct current potential drop and digital image correlation for steel, Int. J. Fatigue 142, 105924 (2021).

[23] ASTM E647-15, Standard Test Method for Measurement of Fatigue Crack Growth Rates (ASTM International, West Conshohocken, PA, 2015).

[24] J. Baró and E. Vives, Analysis of power-law exponents by maximum-likelihood maps, Phys. Rev. E 85, 066121 (2012).

[25] H. Jian, J. Luo, X. Tang, X. Li, and C. Yan, Influence of microstructure on fatigue crack propagation behaviors of an aluminum alloy: Role of sheet thickness, Eng. Fract. Mech. 180, 105 (2017).

[26] Y. Xue, H. El Kadiri, M. Horstemeyer, J. Jordon, and H. Weiland, Micromechanisms of multistage fatigue crack growth in a high-strength aluminum alloy, Acta Mater. 55, 1975 (2007).

[27] Y. Chen, H. Zhang, W. Song, S. Pan, W. Liu, X. Liu, B. Zhu, Y. Song, and W. Zhou, Acceleration effect of a graphite dust environment on the fatigue crack propagation rates of al alloy, Int. J. Fatigue 126, 20 (2019).

[28] A. Shyam and E. Lara-Curzio, A model for the formation of fatigue striations and its relationship with small fatigue crack growth in an aluminum alloy, Int. J. Fatigue 32, 1843 (2010).

[29] D. Kujawski, A fatigue crack driving force parameter with load ratio effects, Int. J. Fatigue 23, 239 (2001).

[30] A. Noroozi, G. Glinka, and S. Lambert, A two parameter driving force for fatigue crack growth analysis, Int. J. Fatigue 27, 1277 (2005).

[31] X. Huang, M. Torgeir, and W. Cui, An engineering model of fatigue crack growth under variable amplitude loading, Int. J. Fatigue 30, 2 (2008).

[32] R. Kokkoniemi, A. Miksic, M. Ovaska, L. Laurson, and M. Alava, Intermittent crack growth in fatigue, J. Stat. Mech. (2017) 073401.

[33] A. Tanguy, M. Gounelle, and S. Roux, From individual to collective pinning: Effect of long-range interaction, Phys. Rev. E 58, 1577 (1998). 